\title{
The undiagnosed disease burden associated with alpha-1 antitrypsin deficiency genotypes
}

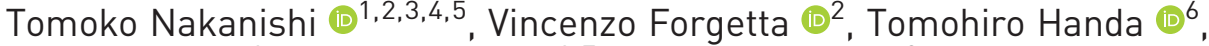 \\ Toyohiro Hirai ${ }^{4}$, Vincent Mooser ${ }^{1,7}$, G. Mark Lathrop ${ }^{8}$, \\ William O.C.M. Cookson (10,10 and J. Brent Richards (10 ${ }^{1,2,11}$
}

Affiliations: ${ }^{1}$ Dept of Human Genetics, McGill University, Montréal, QC, Canada. ${ }^{2}$ Centre for Clinical Epidemiology, Dept of Medicine, Lady Davis Institute for Medical Research, Jewish General Hospital, McGill University, Montréal, QC, Canada. ${ }^{3}$ Kyoto-McGill International Collaborative School in Genomic Medicine, Graduate School of Medicine, Kyoto University, Kyoto, Japan. ${ }^{4}$ Dept of Respiratory Medicine, Graduate School of Medicine, Kyoto University, Kyoto, Japan. ${ }^{5}$ Research Fellow of Japan Society for the Promotion of Science, Tokyo, Japan. ${ }^{6}$ Dept of Advanced Medicine for Respiratory Failure, Graduate School of Medicine, Kyoto University, Kyoto, Japan. ${ }^{7}$ Canada Excellence Research Chair in Genomic Medicine, McGill University, Montréal, QC, Canada. ${ }^{8}$ McGill University and Genome Québec Innovation Centre, Montréal, QC, Canada. ${ }^{9}$ National Heart and Lung Institute, Imperial College London, London, UK. ${ }^{10}$ Royal Brompton and Harefield NHS Foundation Trust, London, UK. ${ }^{11}$ Division of Endocrinology, Depts of Medicine, Human Genetics, Epidemiology and Biostatistics, Jewish General Hospital, McGill University, Montréal, QC, Canada.

Correspondence: J. Brent Richards, Pavillon H-413, Jewish General Hospital, 3755 Cote Ste Catherine, Montréal, QC, Canada, H3T 1E2. E-mail: brent.richardsamcgill.ca

@ERSpublications

Only $6.4 \%$ of those with genotype-defined alpha- 1 antitrypsin deficiency had been diagnosed with this serious disease in UK Biobank. Genotype-guided diagnosis could help to identify the thousands of people in the UK with this partially preventable disease. https://bit.ly/3dMu5Ng

Cite this article as: Nakanishi T, Forgetta V, Handa T, et al. The undiagnosed disease burden associated with alpha-1 antitrypsin deficiency genotypes. Eur Respir J 2020; 56: 2001441 [https://doi.org/10.1183/ 13993003.01441-2020].

ABSTRACT Alpha-1 antitrypsin deficiency (AATD), mainly due to the $\mathrm{PI}^{\star}$ ZZ genotype in SERPINA1, is one of the most common inherited diseases. Since it is associated with a high disease burden and partially prevented by smoking cessation, identification of $\mathrm{PI}^{\star} \mathrm{ZZ}$ individuals through genotyping could improve health outcomes.

We examined the frequency of the $\mathrm{PI}^{\star} \mathrm{ZZ}$ genotype in individuals with and without diagnosed AATD from UK Biobank, and assessed the associations of the genotypes with clinical outcomes and mortality. A phenome-wide association study (PheWAS) was conducted to reveal disease associations with genotypes. A polygenic risk score (PRS) for forced expiratory volume in $1 \mathrm{~s}\left(\mathrm{FEV}_{1}\right) /$ forced vital capacity (FVC) ratio was used to evaluate variable penetrance of $\mathrm{PI}^{\star} \mathrm{ZZ}$.

Among 458164 European-ancestry participants in UK Biobank, 140 had the $\mathrm{PI}^{\star} \mathrm{ZZ}$ genotype and only nine $\left(6.4 \%, 95 \%\right.$ CI 3.4-11.7\%) of them were diagnosed with AATD. Those with $\mathrm{PI}^{\star} \mathrm{ZZ}$ had a substantially higher odds of COPD (OR 8.8, 95\% CI 5.8-13.3), asthma (OR 2.0, 95\% CI 1.4-3.0), bronchiectasis (OR 7.3, 95\%CI 3.2-16.8), pneumonia (OR 2.7, 95\% CI 1.5-4.9) and cirrhosis (OR 7.8, 95\% CI 2.5-24.6) diagnoses and a higher hazard of mortality $\left(2.4,95 \%\right.$ CI 1.2-4.6), compared to $\mathrm{PI}^{\star} \mathrm{MM}$ (wildtype) ( $\mathrm{n}=398$ 424). These associations were stronger among smokers. PheWAS demonstrated associations with increased odds of empyema, pneumothorax, cachexia, polycythaemia, aneurysm and pancreatitis. Polygenic risk score and $\mathrm{PI}^{\star} \mathrm{ZZ}$ were independently associated with $\mathrm{FEV}_{1} / \mathrm{FVC}<0.7$ (OR 1.4 per 1 -sD change, 95\% CI 1.4-1.5 and OR 4.5, 95\% CI 3.0-6.9, respectively).

The important underdiagnosis of AATD, whose outcomes are partially preventable through smoking cession, could be improved through genotype-guided diagnosis.

Copyright CERS 2020. This version is distributed under the terms of the Creative Commons Attribution NonCommercial Licence 4.0. 


\section{Introduction}

Alpha-1 antitrypsin deficiency (AATD) is one of the most common inherited respiratory diseases in people of European descent [1]. Alpha-1 antitrypsin (AAT) is an inhibitor of the proteolytic enzyme elastase and a severe deficiency of AAT enhances the burden of neutrophil elastase in the lungs, leading to emphysema [2]. In addition, intrahepatic accumulation of nonsecreted AAT predisposes to liver diseases [2].

AATD is caused by mutations in the SERPINA1 gene that result in changes in the electrophoretic mobility of the protein predispose to AATD with incomplete penetrance [3, 4]. The most common disease-associated mutation is denoted $\mathrm{PI}^{\star} \mathrm{Z}$ (p.Glu342Lys) and $\mathrm{PI}^{\star} \mathrm{ZZ}$ homozygotes account for the most common phenotype of AATD [2]. The compound heterozygous genotype $\mathrm{PI}^{\star} \mathrm{SZ}$, where $\mathrm{PI}^{\star} \mathrm{S}$ is another missense mutation (p.Val264Glu), is associated with a more mildly increased risk of emphysema in smokers [5]. PIMM refers to homozygosity for wild-type alleles.

AATD is often clinically diagnosed after the identification of COPD or liver disease in individuals with a family history, and the average age at diagnosis is $\sim 45$ years [6]. A previous report, using estimates of allele frequencies from the literature [7], but without direct genotyping, estimated that only 1068 of expected $305009 \mathrm{PI}^{\star} \mathrm{ZZ}$ and $\mathrm{PI}^{\star} \mathrm{SZ}$ individuals had been included in an international AATD registry [8].

Given the partial efficacy of AATD-specific therapies [9] and the availability of smoking cessation counselling, early diagnosis of AATD could promote earlier intervention with smoking cessation therapies and allow for the identification of family members at high risk. Given recent announcements of UK ambitions to sequence 5 million individuals [10], there may exist an opportunity to identify individuals with high-risk genotypes and put in place appropriate diagnostic programmes to reduce the burden of this disease.

Here we sought to understand the prevalence of SERPINA1 genotype status in UK Biobank and assess the diagnosis rate of AATD. We next explored the magnitude of association between SERPINA1 genotypes and respiratory conditions, changes in spirometry results, other extrapulmonary conditions and all-cause mortality. Taking advantage of the large sample size of UK Biobank, we conducted a phenome-wide association study (PheWAS) to investigate potential associations of SERPINA1 genotypes with other outcomes. Lastly, we calculated a polygenic risk score (PRS) for forced expiratory volume in $1 \mathrm{~s}\left(\mathrm{FEV}_{1}\right) /$ forced vital capacity (FVC) ratio to assess the interactions of SERPINA1 genotypes and common variants affecting lung function.

\section{Material and methods \\ UK Biobank study subjects}

UK Biobank is a population-based cohort which recruited people aged 40-69 years from across the UK. We selected 458164 participants of European descent (defined in the supplementary material, figures S1 and S2, table S1) with nonmissing SERPINA1 Z and S genotype information (rs28929474 and rs17580). Both of these variants were genotyped in UK Biobank, and therefore our study is not reliant upon imputation. The minor allele frequencies of rs28929474 and rs17580 were 0.020 and 0.048 , respectively. The genotype definition and the quality control metrics of the genotypes are listed in supplementary table S2. The Z and S allele status of individuals of non-European descent is listed in supplementary table S3.

\section{Ethical compliance}

The UK Biobank was approved by the North West Multi-centre Research Ethics Committee and informed consent was obtained from all participants prior to participation.

\section{Clinical data ascertainment}

Prevalent disease was ascertained by self-reported physician-made diagnoses, self-reported recent medication information for the disease, International Classification of Diseases (ICD)- 9 and -10 codes linked to Hospital Episode Statistics (refer to supplementary table S4 for the specific codes used) available at their initial visit, as in the previous studies of UK Biobank [11, 12]. We acknowledge that common diseases such as COPD and asthma were generally managed in primary care settings, and thus we included self-reported physician-made diagnoses in the disease ascertainment criteria in addition to Hospital Episode Statistics. The UK Biobank study protocol is available online [13]. The curated diagnoses were all known complications of AATD (supplementary table S4) [14-17]. ICD-10 codes in UK Biobank

This article has supplementary material available from erj.ersjournals.com

Received: 27 April 2020 | Accepted after revision: 25 June 2020 
do not have subclassification of AATD by ICD-10 coding (E88.01), but do provide diagnosis of E88.0, which represents the combined diagnoses of plasma-protein metabolism disorders and may include diagnoses, in addition to AATD, such as plasminogen deficiency and bisalbuminaemia. To estimate the prevalence of AATD diagnosis, we identified individuals reporting a physician-made diagnosis of AATD and/or the use of medication for AATD; or having an ICD-9 diagnostic code for AATD. The supplementary material provides the detailed definition of symptoms and the spirometry quality control (supplementary table S5).

\section{Statistical analysis}

Regression models were fitted to assess the associations of SERPINA1 genotypes and clinical outcomes compared to $\mathrm{PI}^{\star} \mathrm{MM}$ genotype. All the models were adjusted for age, sex, genotyping array, assessment centre and the first five principal components in order to account for population structure. We subsequently stratified the participants by smoking status. Within each genotype group, the decrease of $\mathrm{FEV}_{1}$ by age was estimated by linear regression of $\mathrm{FEV}_{1}$ by age, adjusted for the same covariates as above and thus these were not derived by the longitudinal data. Survival analysis was performed using univariate Cox proportional hazard model to estimate the hazard of death. Detailed methods of smoking status definition and survival analysis are presented in the supplementary material (supplementary table S6).

We estimated the national prevalence of $\mathrm{PI}^{\star} \mathrm{ZZ}$ genotype status in the UK, assuming that the allele frequency rates were not different between individuals of European ancestry in UK Biobank and the UK citizens of European ancestry. This could be an underestimate of the $\mathrm{PI}^{\star} \mathrm{ZZ}$ genotype frequency, given that UK Biobank is not a population-representative cohort, as it recruited only those aged $>40$ years and has some healthy volunteer bias. Next, we used data from the Office of National Statistics [18] to estimate the proportion of British citizens of European ancestry and estimated the number of British individuals carrying the $\mathrm{PI}^{\star} \mathrm{ZZ}$ genotype.

\section{Sensitivity analyses}

We included those with E88.0 in ICD-10 codings for the diagnoses of AATD and recalculated the prevalence of the diagnosed AATD. As UK Biobank included pairs of relatives, we removed one randomly selected participant from each pair related to the third degree (kinship coefficient $\geqslant 0.0442$ ), leaving 449991 unrelated participants, to assess the inflation of association affected by familial effects. Multivariate Cox proportional hazard model adjusted for age was also applied for survival analysis.

\section{Phenome-wide association study}

Next, we explored associations of SERPINA1 genotypes with other diseases using a PheWAS design. The detailed methods are described in the supplementary material (supplementary tables S7 and S8).

\section{Polygenic risk score for $\mathrm{FEV}_{1} / \mathrm{FVC}$}

The recent large scale genome-wide association study of spirometry data derived from external cohorts of European descent [19] enabled us to establish a PRS, the weighted sum of effect alleles of common variants that is associated with spirometry results. We calculated the $\mathrm{FEV}_{1} / \mathrm{FVC}$ PRS of each individual and assessed the interactions between SERPINA1 genotypes and this PRS. The detailed methodology is found in the supplementary material.

\section{Results}

We identified 458164 participants in UK Biobank of European descent who had a median age of 58 years (interquartile range (IQR) 50-63 years), and there were $61(0.013 \%)$ people who were diagnosed as having AATD (table 1, supplementary figure S2). Among 140 participants with the PI ZZ genotype, only nine (6.4\%, 95\% CI 3.4-11.7\%) were diagnosed as AATD (table 2). Given that there are 65.6 million citizens of the UK [20], of whom 87\% are estimated to be of European ancestry [18], we estimate that 17439 (95\% CI 14671-20 579) European individuals in the UK carry the PI*ZZ genotype.

Compared to those with $\mathrm{PI}^{\star} \mathrm{MM}$, participants with $\mathrm{PI}^{\star} \mathrm{ZZ}$ had more respiratory symptoms (45\% versus 25\%), lower $\mathrm{FEV}_{1} / \mathrm{FVC}$ (median 0.74 versus 0.77 ) and lower $\mathrm{FEV}_{1} \%$ predicted (median $86 \%$ versus $94 \%$ ) (table 2). A total of 37 (37\%) participants with $\mathrm{PI}^{\star} \mathrm{ZZ}$ had $\mathrm{FEV}_{1} / \mathrm{FVC}<0.7$ (table 2). Among 17790 individuals with a diagnosis of COPD, $31(0.17 \%)$ individuals had the $\mathrm{PI}^{\star} \mathrm{ZZ}$ genotype, and they had more severe airway obstruction than $\mathrm{PI}^{\star} \mathrm{MM}$ individuals. Lastly, only seven (23\%, 95\% CI 11-40\%) $\mathrm{PI}^{\star} \mathrm{ZZ}$ individuals with clinically detected COPD were diagnosed as having AATD (table 3), and among 1407 participants with a diagnosis of cirrhosis, three $(0.21 \%)$ had the $\mathrm{PI}^{\star} \mathrm{ZZ}$ genotype and none of them were diagnosed as AATD. 
TABLE 1 Participant characteristics stratified by SERPINA1 genotype

\begin{tabular}{|c|c|c|c|c|c|c|c|}
\hline & \multirow[t]{2}{*}{ Total } & \multicolumn{6}{|c|}{ Genotype } \\
\hline & & MM & $\mathrm{ZZ}$ & sZ & MZ & SS & MS \\
\hline Subjects & $458164(100)$ & 398424 (87) & $140(0.031)$ & $867(0.19)$ & $16983(3.7)$ & $1013(0.22)$ & 40737 (8.9) \\
\hline Age years & $58(50-63)$ & $58(50-63)$ & $56(49-63)$ & $57(50-63)$ & $58(51-64)$ & $57(50-63)$ & $58(50-63)$ \\
\hline Male & $209694(46)$ & $182344(46)$ & 73 (52) & $393(45)$ & 7715 (45) & $469(46)$ & $18700(46)$ \\
\hline Height cm & $168.7 \pm 9.2$ & $168.6 \pm 9.2$ & $172.2 \pm 9.3$ & $170.1 \pm 9.3$ & $169.6 \pm 9.3$ & $169.1 \pm 9.1$ & $168.8 \pm 9.2$ \\
\hline BMI $\mathrm{kg} \cdot \mathrm{m}^{-2}$ & $27.4 \pm 4.8$ & $27.4 \pm 4.8$ & $26.7 \pm 4.7$ & $27.0 \pm 4.6$ & $27.3 \pm 4.7$ & $27.3 \pm 4.6$ & $27.4 \pm 4.8$ \\
\hline $\begin{array}{l}\text { Subjects with no BMI } \\
\text { data }\end{array}$ & $1522(0.33)$ & $1321(0.33)$ & 0 & $3(0.35)$ & $50(0.29)$ & $4(0.39)$ & $144(0.35)$ \\
\hline Smoking status & 451157 (98) & $392313(98)$ & $135(96)$ & 856 (99) & $16736(99)$ & 996 (98) & $40121(98)$ \\
\hline Current smokers ${ }^{\natural}$ & $47711(11)$ & $41735(11)$ & $7(5.2)$ & $83(9.7)$ & $1605(9.6)$ & $106(11)$ & $4175(10)$ \\
\hline Pack-years & $25.5(14.7-37.8)$ & $25.3(14.7-38.0)$ & $10.3(7.8-14.9)$ & $25.4(16.2-35.0)$ & $24.6(14.3-37.4)$ & $30.0(17.5-39.0)$ & $25.2(14.6-37.5)$ \\
\hline $\begin{array}{l}\text { Subjects with } \\
\text { pack-years data" }\end{array}$ & $103195(65)$ & $89632(65)$ & $27(60)$ & $189(60)$ & $3852(65)$ & $221(66)$ & $9274(65)$ \\
\hline Never-smokers" & $244594(54)$ & $212525(54)$ & $83(61)$ & $460(54)$ & $9216(55)$ & $556(56)$ & $21754(54)$ \\
\hline $\begin{array}{l}\text { Exposure to smoke or } \\
\text { polluted air }\end{array}$ & $120423(26)$ & $104642(26)$ & $36(26)$ & $223(26)$ & 4505 (27) & $256(26)$ & $10761(26)$ \\
\hline AATD diagnosis & $61(0.013)$ & $4(0.0010)$ & $9(6.4)$ & $9(1.0)$ & $36(0.21)$ & 0 & $3(0.0074)$ \\
\hline
\end{tabular}

Data are presented as $n(\%), n$, median (interquartile range) or mean \pm SD. BMI: body mass index; AATD: alpha- 1 antitrypsin deficiency. \#: numbers of individuals were calculated by removing related individuals with kinship coefficients $\geqslant 0.044$, which were used in sensitivity analyses; ${ }^{\text {I: }}$ percentage was calculated among people with information available.

\begin{abstract}
Association of PI*ZZ genotype with clinical outcomes
Those with $\mathrm{P}{ }^{\star} \mathrm{ZZ}$ had a higher risk of COPD (OR 8.8, 95\% CI 5.8-13.3; $\left.\mathrm{p}=1.1 \times 10^{-24}\right)$, asthma (OR 2.0, 95\% CI 1.4-3.0; $\left.\mathrm{p}=5.3 \times 10^{-4}\right)$, bronchiectasis (OR 7.3, 95\% CI 3.2-16.8; $\left.\mathrm{p}=2.4 \times 10^{-6}\right)$ and pneumonia (OR 2.7, $95 \%$ CI 1.5-4.9; $\mathrm{p}=1.2 \times 10^{-3}$ ) compared to $\mathrm{PI}^{\star} \mathrm{MM}$. Those with the $\mathrm{PI}^{\star} \mathrm{ZZ}$ genotype had higher risk of COPD regardless of smoking status, but effect sizes were larger for smokers (OR 13.3, 95\% CI 7.5-23.8
\end{abstract}

TABLE 2 Clinical diagnoses and spirometry results of participants stratified by SERPINA1 genotype

\begin{tabular}{|c|c|c|c|c|c|c|c|c|c|}
\hline & MM & $\mathbf{Z Z}$ & p-value ${ }^{\#}$ & SZ & p-value $\#$ & MZ & p-value ${ }^{\#}$ & SS & p-value ${ }^{\#}$ \\
\hline Subjects & 398424 & 140 & & 867 & & 16983 & & 1013 & \\
\hline Respiratory symptoms & $97970(25)$ & $63(45)$ & $1.8 \times 10^{-7}$ & $219(25)$ & 0.64 & $4150(24)$ & 0.24 & 234 (23) & 0.29 \\
\hline AATD diagnosis & $4(0.0010)$ & $9(6.4)$ & $4.5 \times 10^{-29}$ & $9(1.0)$ & $7.3 \times 10^{-22}$ & $36(0.21)$ & $7.8 \times 10^{-46}$ & 0 & 1 \\
\hline COPD diagnosis & 15502 (3.9) & $31(22)$ & $2.9 \times 10^{-15}$ & $46(5.3)$ & 0.034 & $676(4.0)$ & 0.44 & $36(3.6)$ & 0.68 \\
\hline Asthma diagnosis & $54205(14)$ & $33(24)$ & $1.3 \times 10^{-3}$ & $118(14)$ & 1 & $2343(14)$ & 0.48 & $127(13)$ & 0.34 \\
\hline Bronchiectasis diagnosis & $2767(0.69)$ & $6(4.3)$ & $4.8 \times 10^{-4}$ & $11(1.2)$ & 0.06 & $125(0.74)$ & 0.51 & $10(0.99)$ & 0.25 \\
\hline PFT & 285824 (72) & $101(72)$ & 1 & $613(71)$ & 0.50 & $12110(71)$ & 0.22 & 728 (72) & 0.94 \\
\hline $\mathrm{FEV}_{1} \mathrm{~L}$ & $2.8(2.3-3.3)$ & $2.8(2.1-3.4)$ & 0.28 & $2.8(2.4-3.4)$ & 0.015 & $2.8(2.3-3.4)$ & $3.1 \times 10^{-7}$ & $2.8(2.3-3.3)$ & 0.21 \\
\hline $\mathrm{FEV}_{1} / \mathrm{FVC}$ & $0.77(0.73-0.80)$ & $0.74(0.66-0.79)$ & $3.5 \times 10^{-4}$ & $0.77(0.72-0.81)$ & 0.67 & $0.77(0.73-0.80)$ & 0.63 & $0.77(0.73-0.80)$ & 0.74 \\
\hline $\mathrm{FEV}_{1} \%$ predicted & 94 (83-103) & $86(75-103)$ & $2.3 \times 10^{-4}$ & $95(85-104)$ & 0.28 & $94(84-104)$ & 0.062 & $94(84-104)$ & 0.28 \\
\hline $\begin{array}{l}\text { Decrease in } \mathrm{FEV}_{1} \text { by age } \\
\mathrm{mL} \cdot \mathrm{year}^{-1}\end{array}$ & $35.6(35.4-35.8)$ & $68.4(47.1-89.7)$ & $2.0 \times 10^{-4}$ & $36.0(35.5-37.8)$ & 0.74 & $36.6(35.5-37.8)$ & 0.074 & $34.0(29.4-38.5)$ & 0.51 \\
\hline $\mathrm{FEV}_{1} / \mathrm{FVC}<0.7^{+}$ & $40351(14)$ & 37 (37) & $1.5 \times 10^{-8}$ & $107(17)$ & 0.02 & 1799 (15) & 0.023 & $99(14)$ & 0.75 \\
\hline
\end{tabular}

Data are presented as $\mathrm{n}(\%)$ or median (interquartile range), unless otherwise stated. AATD: alpha-1 antitrypsin deficiency; PFT: pulmonary function testing; $\mathrm{FEV}_{1}$ : forced expiratory volume in $1 \mathrm{~s}$; FVC: forced vital capacity. ${ }^{\#}$ : calculated by comparing to PI*MM genotype; ${ }^{*}$ : estimated with linear regression by age $(95 \% \mathrm{Cl})$ and not derived from the longitudinal data; ${ }^{+}$: percentage calculated among subjects with spirometry information available. 


\begin{tabular}{|c|c|c|c|}
\hline & ZZ & MM & p-value \\
\hline Subjects & $31(0.17)$ & 15502 (95) & \\
\hline Age years & $56(49-63)$ & $62(57-66)$ & 0.47 \\
\hline Male & $21(68)$ & $8016(52)$ & 0.1 \\
\hline Height cm & $173.5 \pm 9.9$ & $167.8 \pm 9.2$ & $1.5 \times 10^{-3}$ \\
\hline No height information & 0 & $63(0.41)$ & 1 \\
\hline BMI $\mathrm{kg} \cdot \mathrm{m}^{-2}$ & $25.8 \pm 4.9$ & $28.4 \pm 5.7$ & $1.9 \times 10^{-3}$ \\
\hline No BMI information & 0 & $100(0.65)$ & 1 \\
\hline Respiratory symptoms & $28(90)$ & $11470(74)$ & 0.04 \\
\hline AATD diagnosis & $7(23)$ & $2(0.013)$ & $2.3 \times 10^{-18}$ \\
\hline Exposure to smoke or polluted air & $10(32)$ & $4173(27)$ & 0.54 \\
\hline Smoking status & 30 (97) & $15159(98)$ & 0.51 \\
\hline Current smokers $\#$ & $2(6.7)$ & $4328(29)$ & $7.1 \times 10^{-3}$ \\
\hline Ex-smokers $\#$ & $19(63)$ & $7249(48)$ & 0.10 \\
\hline Never-smokers $\#$ & $9(30)$ & $3582(24)$ & $4.7 \times 10^{-3}$ \\
\hline PFT & $14(45)$ & $9427(61)$ & 0.095 \\
\hline $\mathrm{FEV}_{1} \mathrm{~L}$ & $1.5(1.7-2.7)$ & $2.2(1.7-2.7)$ & 0.073 \\
\hline $\mathrm{FEV}_{1} / \mathrm{FVC}$ & $0.48(0.42-0.65)$ & $0.70(0.62-0.76)$ & $4.1 \times 10^{-5}$ \\
\hline $\mathrm{FEV}_{1} \%$ predicted & $45(36-79)$ & $76(62-90)$ & $3.8 \times 10^{-3}$ \\
\hline
\end{tabular}

Data are presented as $\mathrm{n}(\%)$, median (interquartile range) or mean $\pm S D$, unless otherwise stated. $\mathrm{n}=17790$. BMI: body mass index; AATD: alpha-1 antitrypsin deficiency; PFT: pulmonary function testing; FEV $_{1}$ : forced expiratory volume in $1 \mathrm{~s}$; FVC: forced vital capacity. " : percentage was calculated among subjects with information on smoking status.

versus OR 7.9, 95\% CI 3.9-16.1). In never-smokers, the $\mathrm{PI}^{\star} \mathrm{ZZ}$ genotype was not significantly associated with asthma or bronchiectasis (figure 1 and supplementary table S9). PI*ZZ was not independently associated with pneumonia when conditioned on the diagnosis of COPD (OR 1.5, 95\% CI 0.8-2.8; $\mathrm{p}=0.21)$.

Among the extrapulmonary diseases we curated, $\mathrm{PI}^{\star} \mathrm{ZZ}$ genotype was associated with diagnoses of cirrhosis (OR 7.8, 95\% CI 2.5-24.6; $\mathrm{p}=0.004$ ), hepatic carcinoma (OR 13.7, 95\% CI 3.4-56.0; $\mathrm{p}=2.7 \times 10^{-4}$ ) and panniculitis (OR 71.8, 95\% CI 9.6-534.9; $\mathrm{p}=3.1 \times 10^{-5}$ ) (supplementary table S9).

Individuals with $\mathrm{PI}^{\star} \mathrm{ZZ}$ had more respiratory symptoms (OR 2.5, 95\% CI $1.8-3.5 ; \mathrm{p}=6.5 \times 10^{-8}$ ) than $\mathrm{PI}^{\star} \mathrm{MM}$, such as wheeze (OR 2.1, 95\% CI 1.5-3.0; $\mathrm{p}=4.0 \times 10^{-5}$ ), shortness of breath (OR 3.3, 95\% CI 1.95.8; $\left.\mathrm{p}=3.0 \times 10^{-5}\right)$, persistent cough (OR 4.2, 95\% CI 2.2-7.8; $\mathrm{p}=9.7 \times 10^{-6}$ ) and persistent sputum (OR 4.1, $95 \%$ CI $\left.2.0-8.2 ; \mathrm{p}=9.1 \times 10^{-5}\right)$. For never-smokers, persistent cough was the only symptom associated with $\mathrm{PI}^{\star} \mathrm{ZZ}$ (OR 3.3, 95\% CI 1.3-8.9; $\mathrm{p}=0.016$ ) (supplementary table S10). People with $\mathrm{PI}^{\star} \mathrm{ZZ}$ genotype were more likely to have $\mathrm{FEV}_{1} / \mathrm{FVC}<0.7$ (OR $4.3,95 \%$ CI $2.8-6.6 ; \mathrm{p}=1.1 \times 10^{-11}$ ) and have $\mathrm{FEV}_{1}<50 \%$ pred (OR 13.2, 95\% CI 6.9-25.5; $\mathrm{p}=1.2 \times 10^{-14}$ ) (figure 1, supplementary table S10). Linear regression of $\mathrm{FEV}_{1}$ by age estimated that the decrease of $\mathrm{FEV}_{1}$ by age is $68.3 \mathrm{~mL} \cdot \mathrm{year}^{-1}$ (95\% CI 47.1-89.7) in $\mathrm{PI}^{*} \mathrm{ZZ}^{1}$ participants compared to $35.6 \mathrm{~mL} \cdot \mathrm{year}^{-1}\left(95 \%\right.$ CI 35.4-35.8) in $\mathrm{PI}^{\star} \mathrm{MM}$ individuals (table 2, supplementary table S10). The difference of the decrease of $\mathrm{FEV}_{1}$ by age between ever-smokers and never-smokers in $\mathrm{PI}^{\star} \mathrm{ZZ}$ individuals was inconclusive because of the lack of statistical power (supplementary table $\mathrm{S} 11$ ). $\mathrm{PI}^{\star} \mathrm{ZZ}$ genotype was associated with all-cause mortality compared to $\mathrm{PI}^{\star} \mathrm{MM}$ genotype (hazard ratio $2.4,95 \%$ CI $1.2-4.6 ; \mathrm{p}=9.9 \times 10^{-3}$ ) during a median follow-up duration of 7.0 years (IQR 6.4-7.7 years) (figure 2, supplementary table S12). All results from sensitivity analyses are presented in the supplement (supplementary tables S12 and S13).

Phenome-wide association study

$\mathrm{PI}^{\star} \mathrm{ZZ}$ genotype was associated with increased risk of other disorders of metabolism (including AATD), emphysema, obstructive chronic bronchitis and chronic airway obstruction (supplementary figure S3). In addition, $\mathrm{PI}^{\star} \mathrm{ZZ}$ was associated with increased risks of dependency on a respirator or supplemental oxygen, empyema and pneumothorax, cachexia, polycythaemia, aneurysm and pancreatitis, all of which were statistically significant $\left(\mathrm{p}<6.1 \times 10^{-4}\right)$ after Benjamini-Hochberg correction in the main analysis, conservatively assuming that all the phecodes tested were independent (supplementary figure S3 and table S14). The more detailed results of sensitivity analyses are in the supplementary material. 


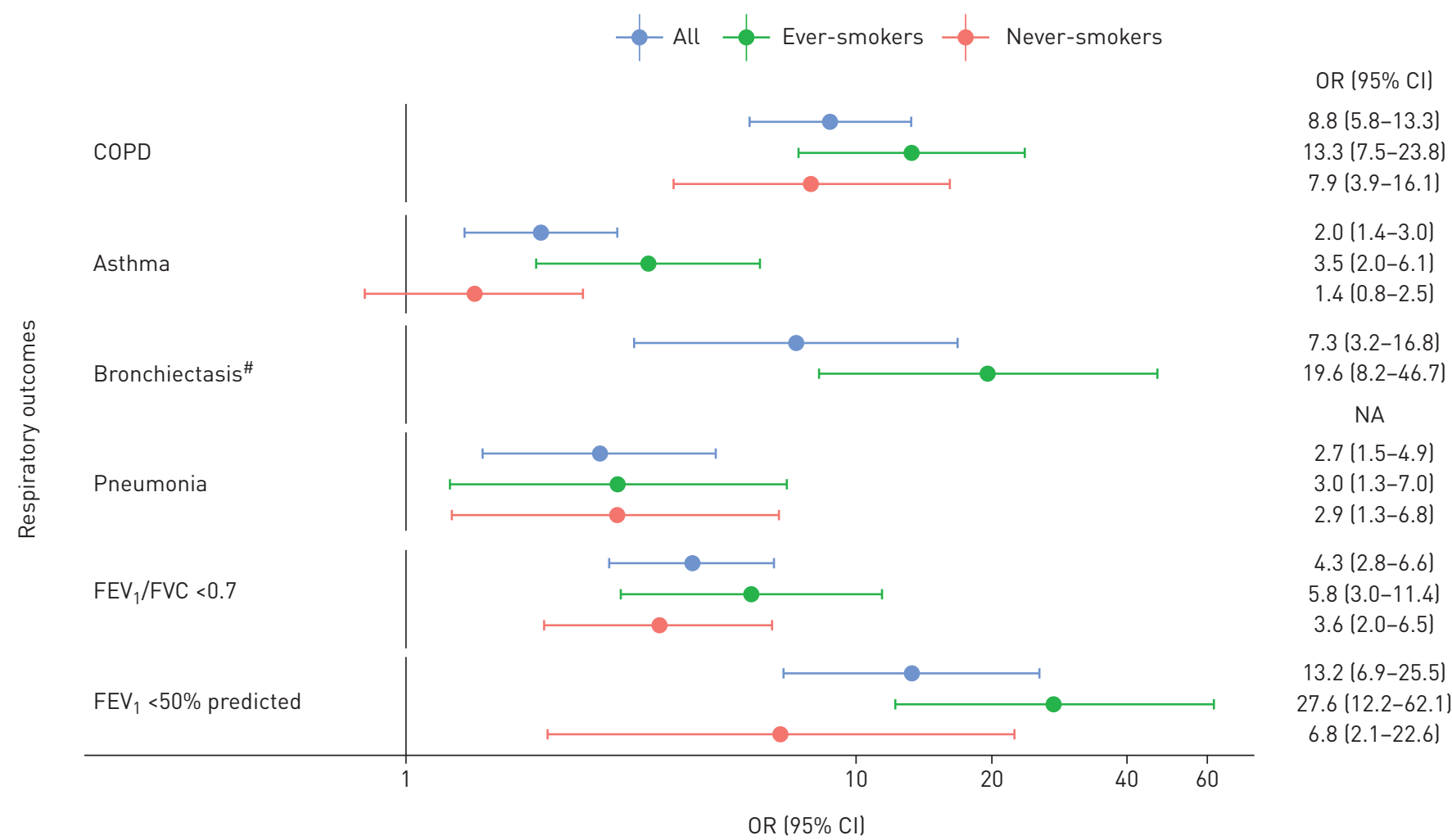

FIGURE 1 Forest plot of associations between the PI*ZZ genotype and prevalent conditions stratified by smoking status. Odds ratios were calculated by logistic regression models compared to the PI*MM (wild-type) genotype adjusted for age, sex, genotyping array, assessment centre and the first five genetic principal components. FEV ${ }_{1}$ : forced expiratory volume in $1 \mathrm{~s}$; FVC: forced vital capacity; NA: not applicable. ${ }^{\#}$ : no neversmokers have been diagnosed with bronchiectasis.

\section{AATD-associated genotypes, other than PI*ZZ}

Additionally, we analysed participants with $\mathrm{PI}^{\star} \mathrm{SZ}, \mathrm{PI}^{\star} \mathrm{MZ}$ and $\mathrm{PI}{ }^{\star} \mathrm{SS}$ compared to $\mathrm{PI}^{\star} \mathrm{MM}$.

In brief, $\mathrm{PI}^{\star} \mathrm{SZ}$ and $\mathrm{PI}^{\star} \mathrm{MZ}$ genotypes were associated with a slight increase of $\mathrm{FEV}_{1} / \mathrm{FVC}<0.7$ (OR 1.3 , 95\% CI 1.0-1.6; $\mathrm{p}=0.022$ and OR 1.1, 95\% CI 1.0-1.1; $\mathrm{p}=0.032$ ), but not associated with increased risk of clinically diagnosed COPD (figure 3, supplementary tables S9 and S10). Among heavy smokers ( $>20$ pack-years), $\mathrm{PI}^{\star} \mathrm{SZ}$ was associated with two-fold increased risk of $\mathrm{FEV}_{1} / \mathrm{FVC}<0.7$ (OR 2.0, 95\% CI 1.3-3.1; $\left.\mathrm{p}=2.6 \times 10^{-3}\right)$, whereas $\mathrm{PI}^{\star} \mathrm{MZ}$ was associated with mildly increased risk of $\mathrm{FEV}_{1} / \mathrm{FVC}<0.7(\mathrm{OR}$ 1.2, 95\% CI 1.1-1.4; $\mathrm{p}=4.5 \times 10^{-4}$ ) (supplementary table S10). $\mathrm{PI}^{\star} \mathrm{MZ}$ was also associated with increased risk of cirrhosis (OR 1.5, 95\% CI 1.2-1.8; $\mathrm{p}=0.002$ ) (figure 3), hepatitis (OR 1.4, 95\% CI 1.1-1.8; $\mathrm{p}=4.6 \times 10^{-3}$ ) and granulomatosis with polyangiitis (OR 2.2, 95\% CI 1.2-3.9; $\mathrm{p}=9.9 \times 10^{-3}$ ) (supplementary table S9). All the other results are provided in the supplementary material (supplementary figures S4 and S5 and tables S9-S12, S15-S17).

\section{Polygenic risk score for FEV $1 / F V C$}

The square of the correlation coefficient $\left(r^{2}\right)$ between observed $\mathrm{FEV}_{1} / \mathrm{FVC}$ and $\mathrm{FEV}_{1} / \mathrm{FVC}$ predicted by the PRS was $3.5 \%(95 \%$ CI $3.4 \%-3.6 \%)$ in the total population $(n=328638)$, which was higher than the correlation between $\mathrm{FEV}_{1} / \mathrm{FVC}$ and smoking status (2.4\%, 95\% CI 2.3\%-2.5\%). The PRS was not associated with other nongenetic risk factors (supplementary table S18). We divided participants into quartiles according to their PRS (figure 4). Among $\mathrm{PI}^{*} \mathrm{ZZ}$ individuals, those with the lowest quartile of PRS, i.e. those at lowest polygenic risk $(n=29)$, had higher $\mathrm{FEV}_{1} / \mathrm{FVC}$ results compared to other $\mathrm{PI}^{\star} \mathrm{ZZ}$ individuals $(\mathrm{n}=72)$ (median (IQR) $0.79(0.67-0.85)$ versus $0.72(0.66-0.77), \mathrm{p}=0.019)$. Multivariate logistic regression indicated that 1 -SD change of $\mathrm{PRS}$ and $\mathrm{PI}^{\star} \mathrm{ZZ}$ are independently associated with $\mathrm{FEV}_{1} / \mathrm{FVC}<0.7$ (OR 1.4, 95\% CI 1.4-1.5; $\mathrm{p}<2 \times 10^{-16}$ and OR 4.5, 95\% CI 3.0-6.9; $\mathrm{p}=2.3 \times 10^{-12}$, respectively) (supplementary table S19).

\section{Discussion}

Undertaking a large-scale assessment of the prevalence of SERPINA1 genotypes, their associated odds of morbidity and mortality and the diagnostic rates of AATD in UK Biobank, we found that the vast 

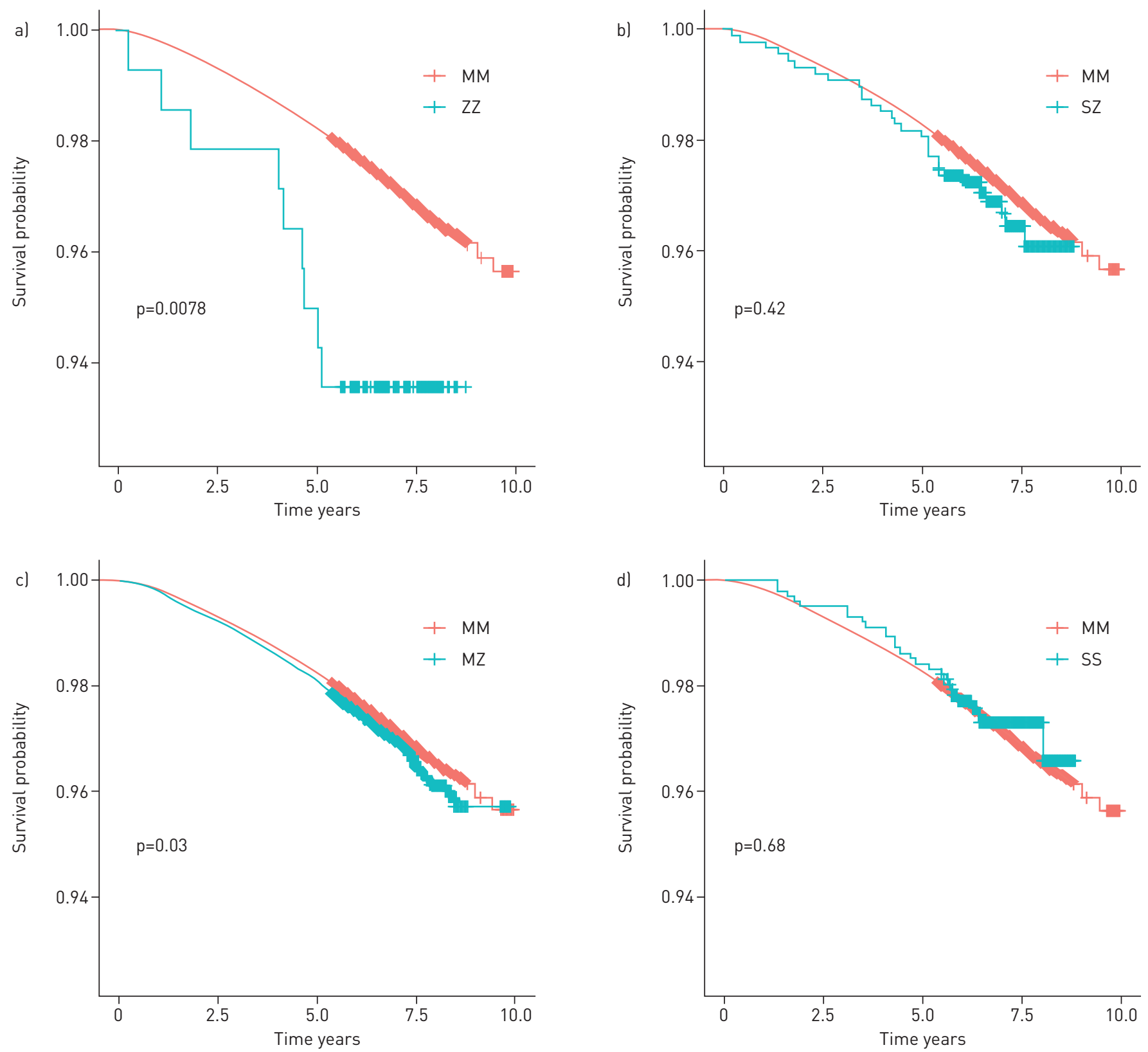

FIGURE 2 Survival curves of all-cause mortality stratified by SERPINA1 genotypes. a) PI*ZZ versus PI*MM genotypes; b) PI*SZ versus PI*MM genotypes; c) PI*MZ versus $\mathrm{PI}$ *MM genotypes; d) PI*SS versus $\mathrm{PI} M \mathrm{MM}$ genotypes. All p-values were calculated by log-rank test.

majority of individuals with $\mathrm{PI}^{\star} \mathrm{ZZ}$ were not diagnosed as having AATD. Yet, these individuals had substantially increased odds of respiratory symptoms, diseases and all-cause mortality. We estimated that $\sim 17000$ individuals in the UK carry the $\mathrm{PI}^{\star} \mathrm{ZZ}$ genotype, which was similar to the estimates from the prior population-based neonatal screening studies [21, 22]. Nevertheless, this could be an underestimate given that UK Biobank recruited only those aged $>40$ years, and very ill individuals are unlikely to be able to take part. Thus, while the proportion of all British individuals who could be detected through genotyping efforts is small, the absolute number is not.

The impact of $\mathrm{PI}^{\star} \mathrm{ZZ}$ genotype on health status is striking. $\mathrm{PI}^{\star} \mathrm{ZZ}$ was associated with increased risk of COPD and pneumonia regardless of smoking status, yet the effect sizes for COPD were substantially larger among smokers. Furthermore, $\mathrm{PI}^{\star} \mathrm{ZZ}$ genotype was associated with increased risk of asthma and bronchiectasis only among smokers. This suggests that smoking cessation has the potential to prevent those with $\mathrm{PI}^{\star} \mathrm{ZZ}$ genotype from developing multiple respiratory diseases.

Almost half of those with the $\mathrm{PI}^{\star} \mathrm{ZZ}$ genotype were symptomatic with severe airflow obstruction and increased risk of all-cause mortality. Linear regression of $\mathrm{FEV}_{1}$ by age in $\mathrm{PI}^{\star} \mathrm{ZZ}$ individuals estimated a 


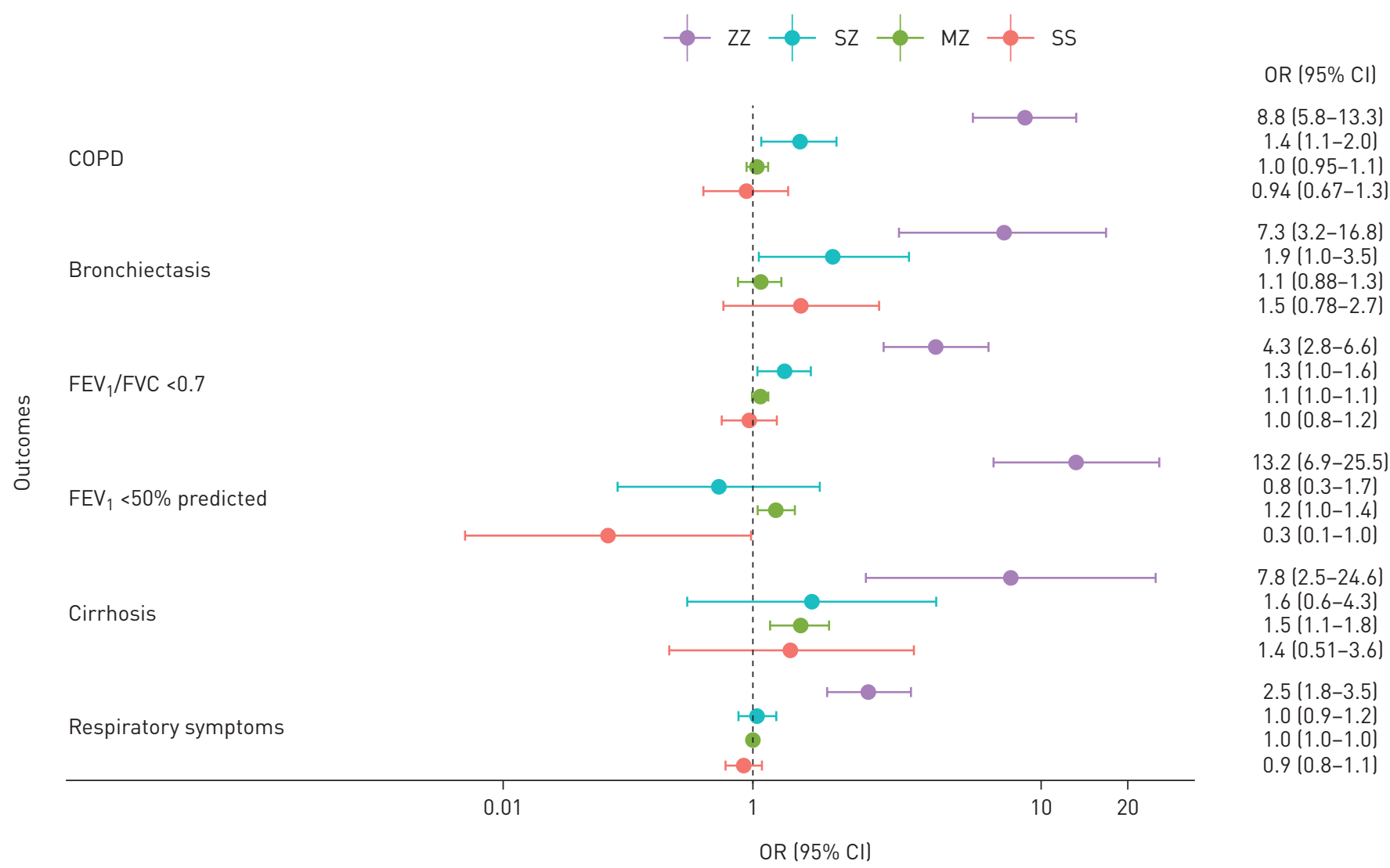

FIGURE 3 Forest plot of associations between SERPINA1 genotypes and common conditions. Odds ratios were calculated by logistic regression models compared to PI*MM (wild-type) genotype adjusted for age, sex, genotyping array, assessment centre and the first five genetic principal components. $\mathrm{FEV}_{1}$ : forced expiratory volume in $1 \mathrm{~s}$; FVC: forced vital capacity.

larger age-dependent decrease of $\mathrm{FEV}_{1}$ compared to $\mathrm{PI}^{\star} \mathrm{MM}$ individuals. In $\mathrm{PheWAS}, \mathrm{PI}^{\star} \mathrm{ZZ}$ was significantly associated with dependence on a respirator or supplemental oxygen, empyema and pneumothorax, cachexia and secondary polycythaemia, all of which could be sequelae of AATD. Extrapulmonary diseases that have previously been described as associated with $\mathrm{PI}^{*} \mathrm{ZZ}$ were also replicated in our study, such as cirrhosis, hepatic carcinoma, panniculitis, pancreatitis and aneurysm, pathogenesis of which is thought to be triggered by protease-antiprotease imbalance [23].

Even among subjects with COPD diagnosis, $77 \%$ of $\mathrm{PI}^{\star} \mathrm{ZZ}$ individuals were not diagnosed as having AATD in this study. Previous surveys indicated that the mean delay between symptom onset and diagnosis among those actually diagnosed ranges from 5 to 8 years $[6,24]$, and the delay was associated with worse respiratory symptoms and accelerated emphysema progression [25]. Potential reasons for underdiagnosis include poor awareness of the disease, the unavailability of appropriate tests and/or treatments in specific regions, i.e. no availability of AAT replacement therapy in the UK $[26,27]$. The current laboratory testing practice for AATD involves first quantifying plasma AAT levels together with measuring C-reactive protein, followed by protein phenotyping and/or $\mathrm{Z}$ and $\mathrm{S}$ genotyping $[28,29]$. Since the genotype data is less affected by batch effects compared to measuring AAT, a protein known to increase in the context of inflammatory conditions [30], our results suggest that genotyping could be a step toward efficient identification of $\mathrm{PI}^{\star} \mathrm{ZZ}$ carriers. In the current study, $80 \%$ of diagnosed AATD occurred in $\mathrm{PI}^{\star} \mathrm{MZ}^{*}$ individuals. This could reflect either misdiagnoses or the impact of other disease predisposing mutations in the SERPINA1 gene that were not detected with the genotyping array.

$\mathrm{PI}^{\star} \mathrm{ZZ}$ individuals with the lowest quartile of the PRS had relatively higher $\mathrm{FEV}_{1} / \mathrm{FVC}$, possibly suggesting that polygenic factors affecting lung function partially explain variable penetrance of $\mathrm{PI}^{\star} \mathrm{ZZ}$ genotype [3]. Genome-wide genotyping, which enables the calculation of the PRS and the SERPINA1 genotyping, could be alternative approach to the SERPINA1-targeted genotyping as a screening strategy for AATD, given its relatively low cost (USD 40 in a research context).

In addition, our study provides several insights of the effects of $\mathrm{PI}^{\star} \mathrm{SZ}$ and $\mathrm{PI}^{\star} \mathrm{MZ}$ genotype. Overall, while $\mathrm{PI}^{\star} \mathrm{SZ}$ was associated with a two-fold increased risk of an $\mathrm{FEV}_{1} / \mathrm{FVC}<0.7$ in heavy smokers, we 


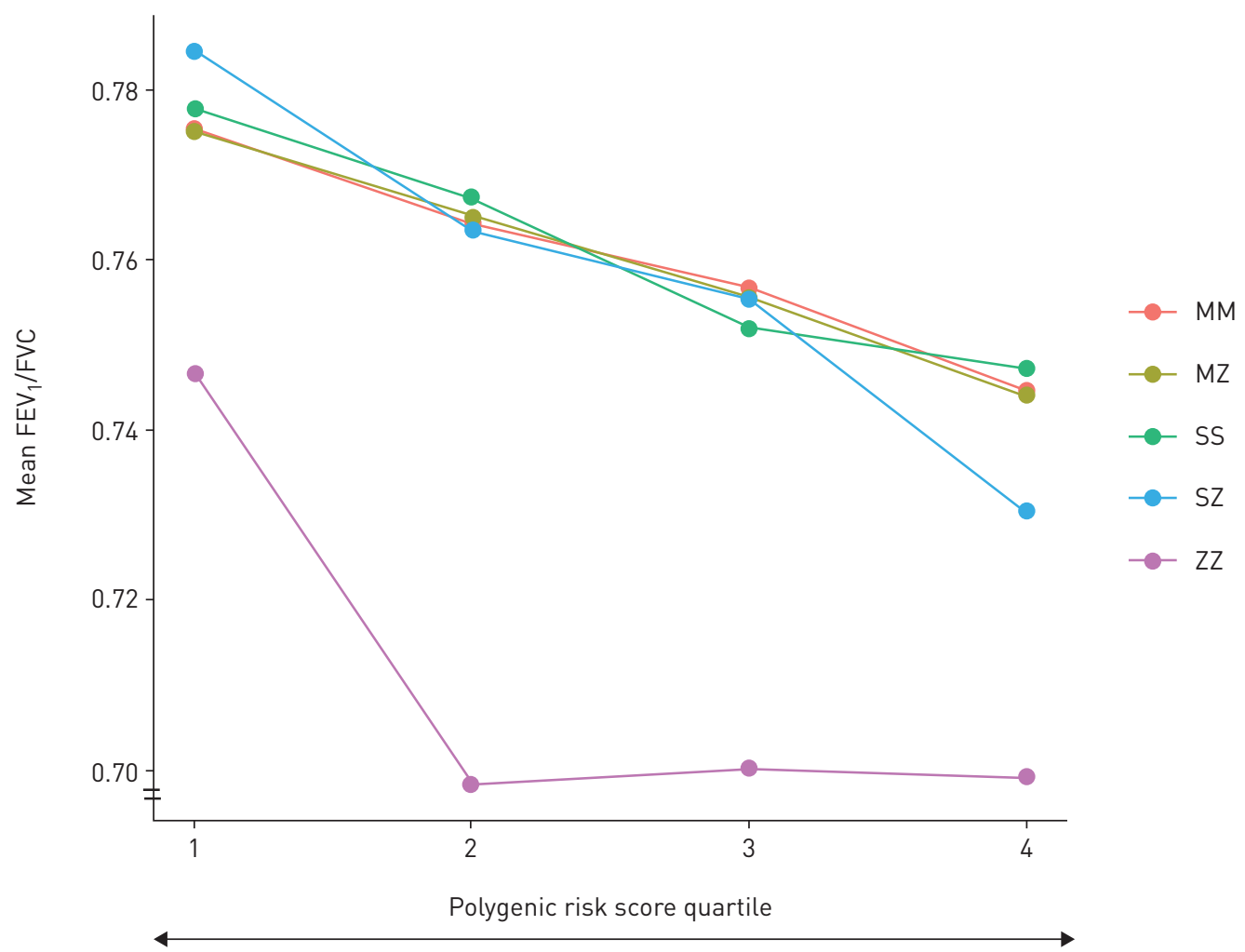

Predicted high $\mathrm{FEV}_{1} / \mathrm{FVC}$

Predicted low $\mathrm{FEV}_{1} / \mathrm{FVC}$

FIGURE 4 Mean of observed forced expiratory volume in $1 \mathrm{~s}\left(\mathrm{FEV}_{1}\right) /$ forced vital capacity $(\mathrm{FVC})$ stratified by polygenic risk score quartile. Polygenic risk scores were calculated by LDpred using genome-wide association study summary statistics for $\mathrm{FEV}_{1} / \mathrm{FVC}$ derived from the SpiroMeta consortium, which consists of individuals of European descent. Detailed methods are described in the supplementary material.

demonstrated that $\mathrm{PI}^{\star} \mathrm{SZ}$ and $\mathrm{PI}^{\star} \mathrm{MZ}$ genotypes had modest effects on the risks of spirometry-defined obstructive lung impairment $\left(\mathrm{FEV}_{1} / \mathrm{FVC}<0.7\right)$ and severe airways obstruction $\left(\mathrm{FEV}_{1}<50 \%\right.$ pred) compared to the previous findings that $\mathrm{PI}^{\star} \mathrm{SZ}$ had three-fold increased risk of COPD (95\% CI 1.24-8.57) [5] and $\mathrm{PI}^{\star} \mathrm{MZ}$ had five-fold increased risk of COPD (95\% CI 1.27-21.15) [31]. However, these casecontrol studies described very large confidence intervals and the $\mathrm{PI}^{\star} \mathrm{MZ}$ participants were recruited from index $\mathrm{PI}^{\star} \mathrm{MZ}$ COPD patients [31], potentially biased by the other shared genetic factors associated with COPD. PI* MZ genotype, but not $\mathrm{PI}^{\star} \mathrm{SZ}$, was significantly associated with increased risk of cirrhosis and marginally increased risk of all-cause mortality in this study. The discordance between $\mathrm{PI}^{\star} \mathrm{SZ}$ and $\mathrm{PI}^{\star} \mathrm{MZ}$ genotype could be driven by the lack of statistical power in $\mathrm{PI}^{\star} \mathrm{SZ}$ individuals, 20 times less than $\mathrm{PI}^{\star} \mathrm{MZ}$. PheWAS found that $\mathrm{PI}^{\star} \mathrm{MZ}$ was associated with multiple diseases, namely increased risk of cholelithiasis and decreased risk of cardiovascular disease. There are several studies [32-35] which might support these hypotheses, although validation studies and functional investigations are necessary.

Most of the previous epidemiological studies of $\mathrm{PI}^{\star} \mathrm{ZZ}$ individuals were case-control studies [36, 37] and the current study is the one of the largest studies to assess the effects of the SERPINA1 genotype status to multiple health conditions in a single large population cohort. A prior family-based study included nonindex family members with undiagnosed $\mathrm{PI}^{\star} \mathrm{ZZ}$ individuals who had more severe spirometry results (mean $\mathrm{FEV}_{1} / \mathrm{FVC} 0.61$ and mean $\mathrm{FEV}_{1} 72.3 \%$ pred) [38] than those in UK Biobank (table 2), which could reflect the effects of other shared genetic factors. The main limitation of this study is that UK Biobank is not representative of general population as there is well-documented evidence of a "healthy volunteer' bias [39]. Therefore, we did not try to derive generalisable disease prevalence, but aimed to report the associations with $\mathrm{PI}^{\star} \mathrm{ZZ}$ genotype and multiple health conditions. Another shortcoming is that the diagnosis of AATD was based on questionnaires and/or Hospital Episode Statistics, which rely on the diagnosis of each clinician and potentially harbour "clinical order" bias [40]. Nevertheless, the estimated prevalence of asthma (14\%), COPD (3.9\%) and bronchiectasis (0.69\%) in $\mathrm{PI}^{*} \mathrm{MM}$ individuals were similar to the previous reports [41-43], which might support the validity of our approach of how to ascertain the disease status. PheWAS demonstrated that $\mathrm{PI}^{\star} \mathrm{ZZ}$ was associated with increased risk of cystic fibrosis, 
which could represent misdiagnoses of bronchiectasis. PheWAS, which is based on ICD codings, can be underpowered, so that while no significant associations between $\mathrm{PI}^{\star} \mathrm{ZZ}$ and liver diseases or asthma were observed, this does not preclude smaller effects. Last, there are no AAT measurements available in UK Biobank, so we could not test whether people with high-risk genotypes had low levels of plasma AAT. Although we did not test cost-effectiveness of the population-level screening of AATD, genome-wide genotyping may help the screening of individuals at risk, such as heavy smokers or with a family history of pulmonary disease, to identify those with undiagnosed AATD. As this is a genetic study with potential clinical implications, future effort is needed to address the issue of incidental findings, such as applying the American College of Medical Genetics and Genomics [44] recommendations as to how to report secondary findings.

In summary, we provide evidence that the vast majority of individuals with $\mathrm{PI}^{\star} \mathrm{ZZ}$ are not diagnosed as having AATD, according to definitions available in UK Biobank. Yet these individuals have a profoundly increased burden of multiple symptoms and diseases and an increased risk of all-cause mortality. Identification of these individuals could help to target smoking cessation programmes [45] and the ascertainment of family members, as well as disease-specific therapies [9]. Our data provide potential avenues to realise clinical benefits of emerging nationwide genomic efforts in the UK.

Acknowledgements: We appreciate the individuals who participated in UK Biobank.

Author contributions: Conception and design: T. Nakanishi and J.B. Richards. Data analyses: T. Nakanishi. Data acquisition: T. Nakanishi, V. Forgetta and J.B. Richards. Interpretation of data: T. Nakanishi, V. Forgetta, T. Handa, T. Hirai, V. Mooser, G.M. Lathrop, W.O.C.M. Cookson and J.B. Richards. Intellectual contribution to the manuscript: T. Nakanishi, V. Forgetta, T. Handa, T. Hirai, V. Mooser, G.M. Lathrop, W.O.C.M. Cookson and J.B. Richards. All authors were involved in preparation of the further draft of the manuscript and revising it critically for content. All authors gave final approval of the version to be published. T. Nakanishi and J.B. Richards are the guarantors. The corresponding author attests that all listed authors meet authorship criteria and that no others meeting the criteria have been omitted.

Conflict of interest: T. Nakanishi has nothing to disclose. V. Forgetta has nothing to disclose. T. Handa is in the employ of the Collaborative Research Laboratory funded by Teijin Pharma Co., Ltd. T. Hirai reports grants from The Intractable Respiratory Diseases and Pulmonary Hypertension Research Group, the Ministry of Health, Labor and Welfare, Japan, outside the submitted work. V. Mooser has nothing to disclose. G.M. Lathrop has nothing to disclose. W.O.C.M. Cookson has nothing to disclose. J.B. Richards has nothing to disclose.

Support statement: T. Nakanishi is supported by Research Fellowships of Japan Society for the Promotion of Science (JSPS) for Young Scientists and JSPS Overseas Challenge Program for Young Researchers. J.B. Richards is supported by Canadian Institutes of Health Research (CIHR), the Fonds de Recherche Québec Santé (FRQS) Clinical Research Scholarship. T. Handa is supported by JSPS (17K09612). T. Hirai is supported by a grant to The Intractable Respiratory Diseases and Pulmonary Hypertension Research Group, the Ministry of Health, Labor and Welfare, Japan. The funding sources had no role in: the design or conduct of the study; the collection, management, analysis, and interpretation of the data; or the preparation, review, or approval of the manuscript. The Richards research group is supported by CIHR, the Lady Davis Institute of the Jewish General Hospital and FRQS. This research has been conducted using the UK Biobank Resource (project number: 27449). Funding information for this article has been deposited with the Crossref Funder Registry.

\section{References}

Silverman EK, Sandhaus RA. Alpha1-antitrypsin deficiency. N Engl J Med 2009; 360: 2749-2757.

Stoller JK, Aboussouan LS. A review of $\alpha 1$-antitrypsin deficiency. Am J Respir Crit Care Med 2012; 185: 246-259.

Silverman EK, Pierce JA, Province MA, et al. Variability of pulmonary function in alpha-1-antitrypsin deficiency: clinical correlates. Ann Intern Med 1989; 111: 982-991.

4 DeMeo DL, Campbell EJ, Brantly ML, et al. Heritability of lung function in severe alpha-1 antitrypsin deficiency. Hum Hered 2009; 67: 38-45.

5 Dahl M, Hersh CP, Ly NP, et al. The protease inhibitor PIS allele and COPD: a meta-analysis. Eur Respir J 2005; 26: $67-76$.

6 Campos MA, Wanner A, Zhang G, et al. Trends in the diagnosis of symptomatic patients with $\alpha 1$ - antitrypsin deficiency between 1968 and 2003. Chest 2005; 128: 1179-1186.

7 De Serres FJ. Worldwide racial and ethnic distribution of $\alpha_{1}$-antitrypsin deficiency: summary of an analysis of published genetic epidemiologic surveys. Chest 2002; 122: 1818-1829.

8 Luisetti M, Seersholm N. $\alpha_{1}$-Antitrypsin deficiency 1: epidemiology of $\alpha_{1}$-antitrypsin deficiency. Thorax 2004; 56: 164-169.

9 Chapman KR, Burdon JGW, Piitulainen E, et al. Intravenous augmentation treatment and lung density in severe $\alpha 1$ antitrypsin deficiency (RAPID): a randomised, double-blind, placebo-controlled trial. Lancet 2015; 386: 360-368.

10 Turro E, Astle W, Megy K. Whole-genome sequencing of rare disease patients in a national healthcare system. Nature 2020; 583: 96-102.

11 Said MA, Verweij N, van der Harst P. Associations of combined genetic and lifestyle risks with incident cardiovascular disease and diabetes in the UK Biobank study. JAMA Cardiol 2018; 3: 693-702.

12 Pilling LC, Tamosauskaite J, Jones G, et al. Common conditions associated with hereditary haemochromatosis genetic variants: cohort study in UK Biobank. BMJ 2019; 364: k5222. 
13 UK Biobank. 2007. UK Biobank: Protocol for a large-scale prospective epidemiological resource. www.ukbiobank. ac.uk/wp-content/uploads/2011/11/UK-Biobank-Protocol.pdf.

14 Eriksson S, Carlson J, Velez R. Risk of cirrhosis and primary liver cancer in alphal-antitrypsin deficiency. $N$ Engl J Med 1986; 314: 736-739.

15 McBean J, Sable A, Maude J, et al. Alpha1-antitrypsin deficiency panniculitis. Cutis 2003; 71: $205-209$.

16 Mahr AD, Edberg JC, Stone $\mathrm{JH}$, et al. Alpha $\mathrm{A}_{1}$-antitrypsin deficiency-related alleles $\mathrm{Z}$ and $\mathrm{S}$ and the risk of Wegener's granulomatosis. Arthritis Rheum 2010; 62: 3760-3767.

17 Sun Z, Yang P. Role of imbalance between neutrophil elastase and $\alpha 1$-antitrypsin in cancer development and progression. Lancet Oncol 2004; 5: 182-190.

18 Office for National Statistics (ONS). Key Statistics and Quick Statistics for Local Authorities in the United Kingdom. Newport, Office for National Statistics, 2013; pp. 1-27.

19 Shrine N, Guyatt AL, Erzurumluoglu AM, et al. New genetic signals for lung function highlight pathways and chronic obstructive pulmonary disease associations across multiple ancestries. Nat Genet 2019; 51: 481-493.

20 Office for National Statistics (ONS). National Population Projections: 2016-based Statistical Bulletin. 2017. www. ons.gov.uk/peoplepopulationandcommunity/populationandmigration/populationprojections/bulletins/nationalpopu lationprojections/2016basedstatisticalbulletin

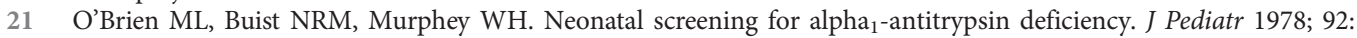
$1006-1010$

22 Sveger T. Liver disease in alphal-antitrypsin deficiency detected by screening of 200,000 infants. N Engl J Med 1976; 294: 1316-1321.

23 Wang Q, Du J, Yu P, et al. Hepatic steatosis depresses alpha-1-antitrypsin levels in human and rat acute pancreatitis. Sci Rep 2015; 5: 17833.

24 Stoller JK, Sandhaus RA, Turino G, et al. Delay in diagnosis of $\alpha_{1}$-antitrypsin deficiency: a continuing problem. Chest 2005; 128: 1989-1994.

25 Tejwani V, Nowacki AS, Fye E, et al. The impact of delayed diagnosis of alpha-1 antitrypsin deficiency: the association between diagnostic delay and worsened clinical status. Respir Care 2019; 64: 915-922.

26 Greulich T, Vogelmeier CF. Alpha-1-antitrypsin deficiency: increasing awareness and improving diagnosis. Ther Adv Respir Dis 2016; 10: 72-84.

27 Horváth I, Canotilho M, Chlumský J, et al. Diagnosis and management of $\alpha_{1}$-antitrypsin deficiency in Europe: an expert survey. ERS Open Res 2019; 5: 00171-2018.

28 Miravitlles M, Dirksen A, Ferrarotti I, et al. European Respiratory Society statement: Diagnosis and treatment of pulmonary disease in $\alpha_{1}$-antitrypsin deficiency. Eur Respir J 2017; 50: 1700610.

29 Franciosi AN, Carroll TP, McElvaney NG. Pitfalls and caveats in $\alpha 1$-antitrypsin deficiency testing: a guide for clinicians. Lancet Respir Med 2019; 7: 1059-1067.

30 Sanders CL, Ponte A, Kueppers F. The effects of inflammation on alpha 1 antitrypsin levels in a national screening cohort. COPD 2018; 15: 10-16.

31 Molloy K, Hersh CP, Morris VB, et al. Clarification of the risk of chronic obstructive pulmonary disease in $\alpha_{1}$-antitrypsin deficiency PiMZ heterozygotes. Am J Respir Crit Care Med 2014; 189: 419-427.

32 Fadini GP, Menegazzo L, Rigato M, et al. NETosis delays diabetic wound healing in mice and humans. Diabetes 2016; 65: 1061-1071.

33 Dijk W, Kersten S. Regulation of lipoprotein lipase by Angptl4. Trends Endocrinol Metab 2014; 25 : 146-155.

34 Ferkingstad E, Oddsson A, Gretarsdottir S, et al. Genome-wide association meta-analysis yields 20 loci associated with gallstone disease. Nat Commun 2018; 9: 5101.

35 Fähndrich S, Biertz F, Karch A, et al. Cardiovascular risk in patients with alpha-1-antitrypsin deficiency. Respir Res 2017; 18: 171.

36 Piitulainen E, Eriksson S. Decline in $\mathrm{FEV}_{1}$ related to smoking status in individuals with severe $\alpha_{1}$-antitrypsin deficiency (PiZZ). Eur Respir J 1999; 13: 247-251.

37 Tanash HA, Nilsson PM, Nilsson JÅ, et al. Clinical course and prognosis of never-smokers with severe alpha-1-antitrypsin deficiency (PiZZ). Thorax 2008; 63: 1091-1095.

38 DeMeo DL, Sandhaus RA, Barker AF, et al. Determinants of airflow obstruction in severe alpha-1-antitrypsin deficiency. Thorax 2007; 62: 805-812.

39 Fry A, Littlejohns TJ, Sudlow C, et al. Comparison of sociodemographic and health-related characteristics of UK Biobank participants with those of the general population. Am J Epidemiol 2017; 186: 1026-1034.

40 Bush WS, Oetjens MT, Crawford DC. Unravelling the human genome-phenome relationship using phenome-wide association studies. Nat Rev Genet 2016; 17: 129-145.

41 Health and Social Care Information Centre. Health Survey for England 2018 Asthma. 2019. healthsurvey.hscic.gov. uk/media/81643/HSE18-Asthma-rep.pdf Date last updated: December 3, 2019. Date last accessed: June 4, 2020.

42 Health and Safety Executive (HSE). 2019. Work-related Chronic Obstructive Pulmonary Disease (COPD) statistics in Great Britain, 2019. https://www.hse.gov.uk/statistics/causdis/copd.pdf.

43 Quint JK, Millett ERC, Joshi M, et al. Changes in the incidence, prevalence and mortality of bronchiectasis in the UK from 2004 to 2013: a population-based cohort study. Eur Respir J 2016; 47: 186-193.

44 Kalia SS, Adelman K, Bale SJ, et al. Recommendations for reporting of secondary findings in clinical exome and genome sequencing, 2016 update (ACMG SF v2.0): a policy statement of the American College of Medical Genetics and Genomics. Genet Med 2017; 19: 249-255.

45 Seersholm N, Kok-Jensen A. Survival in relation to lung function and smoking cessation in patients with severe hereditary alpha 1-antitrypsin deficiency. Am J Respir Crit Care Med 1995; 151: 369-373. 